Mathematical Modelling and Analysis

Volume 20 Number 3, May 2015, 369-381

http://dx.doi.org/10.3846/13926292.2015.1048759

(c) Vilnius Gediminas Technical University, 2015
Publisher: Taylor\&Francis and VGTU

http://www.tandfonline.com/TMMA

ISSN: $1392-6292$

eISSN: 1648-3510

\title{
Solving Boundary Value Problems for Second Order Singularly Perturbed Delay Differential Equations by $\varepsilon$-Approximate Fixed-Point Method
}

\author{
Zbigniew Bartoszewski ${ }^{a}$ and Anna Baranowska ${ }^{b}$ \\ ${ }^{a}$ Faculty of Applied Physics and Mathematics, Gdańsk University of \\ Technology \\ Narutowicza 11/12, 80-233 Gdańsk, Poland \\ ${ }^{b}$ Institute of Oceanology, Polish Academy of Sciences \\ Powstańców Warszawy 55, 81-712 Sopot, Poland \\ E-mail(corresp.): zbart@mifgate.mif.pg.gda.pl \\ E-mail: anbar@iopan.gda.pl
}

Received October 1, 2014; revised April 10, 2015; published online May 15, 2015

\begin{abstract}
In this paper, the boundary value problem for second order singularly perturbed delay differential equation is reduced to a fixed-point problem $v=\mathcal{A} v$ with a properly chosen (generally nonlinear) operator $\mathcal{A}$. The unknown fixed-point $v$ is approximated by cubic spline $v_{h}$ defined by its values $v_{i}=v_{h}\left(t_{i}\right)$ at grid points $t_{i}, i=0,1, \ldots, N$. The necessary for construction the cubic spline and missing the first derivatives at the boundary are replaced by the derivatives of the corresponding interpolating polynomials matching the grid points values nearest to the boundary points. An approximation of the solution is obtained by minimization techniques applied to a function whose arguments are the grid point values of the sought spline. The results of numerical experiments with two boundary value problems for the second order singularly perturbed delay differential equations as well as their comparison with the results of other methods employed by other authors are also provided.
\end{abstract}

Keywords: singularly perturbation problems, delay differential equations, fixed-point method, cubic splines, absolute errors.

AMS Subject Classification: 34B99; 65L11; 65L20.

\section{Introduction}

The problems we consider in this paper are boundary value problems for second order singularly perturbed delay differential equations of the form

$$
\begin{aligned}
& \epsilon y^{\prime \prime}(x)=f\left(x, y(x), y^{\prime}(x), y(\alpha(x))\right), \quad a \leq x \leq b, \\
& y(x)=\phi(x) \quad \text { for } x \leq a, \quad y(b)=\psi,
\end{aligned}
$$


where the functions $f, \phi$ and $\alpha$,

$$
\begin{aligned}
& f: D \rightarrow \mathbb{R}, \quad D=\left\{\left(t, z_{1}, z_{2}, z_{3}\right): a \leq t \leq b,-\infty \leq z_{i} \leq+\infty\right\}, \\
& \phi:[\gamma, a] \rightarrow \mathbb{R}, \quad \alpha:[a, b] \rightarrow(-\infty, b], \quad \gamma=\min _{a \leq x \leq b} \alpha(x)
\end{aligned}
$$

are continuous and $0<\epsilon \ll 1$. Without a loss of generality we can assume that $y(a)=y(b)=0$.

The singularly perturbed problems appear in many areas of science and technology and there exists a vast literature devoted to them. A good and comprehensive review of the literature related to singularly perturbed problems, perturbation methods and their applications can be found in [9], where the bibliography consists of 513 items and among which there are research papers, books devoted to the general theory and different applications, survey papers and conference proceedings related to singularly perturbed problems. So, we refer all the readers interested in the subject to this paper.

A method of solution of problem (1.1)-(1.2) we will propose was already presented in [2] as an approximate method of solution of boundary value problems for delay differential equations (DDE in short) of the form

$$
\begin{aligned}
& y^{\prime \prime}(x)=f\left(x, y(x), y^{\prime}(x), y(\alpha(x))\right), \quad a \leq x \leq b, \\
& y(x)=\phi(x) \quad \text { for } \min _{x \in[a, b]} \alpha(x) \leq x \leq a, \quad y(b)=0,
\end{aligned}
$$

where the functions $f, \alpha$ and $\phi$,

$$
\begin{aligned}
& f: D \rightarrow \mathbb{R}, \quad D=\left\{\left(t, z_{1}, z_{2}, z_{3}\right): a \leq t \leq b,-\infty \leq z_{i} \leq+\infty\right\}, \\
& \alpha:[a, b] \rightarrow[\beta, b], \quad \beta \leq a, \quad \phi:[\beta, a] \rightarrow \mathbb{R},
\end{aligned}
$$

are continuous and $\phi(a)=0$.

It was also discussed in [3] as an approximate method of solution of more general boundary value problems for the system of DDEs of the form

$$
\begin{aligned}
& \frac{d y(t)}{d t}=f\left(t, y(t), y\left(t-\tau_{1}\right), \ldots, y\left(t-\tau_{k}\right)\right), \quad t \in(a, b], \\
& P_{1} y(t)+P_{2} y(b-a+t)=\varphi(t), \quad t \in[a-\tau, a],
\end{aligned}
$$

where $f=\left(f_{1}, \ldots, f_{n}\right)^{T}:[a, b] \times \mathbb{R}^{k+1} \rightarrow \mathbb{R}^{n}$ is continuous on some compact subset $\bar{D}$ of the set $[a, b] \times \mathbb{R}^{k+1}, y(t)=\left(y_{1}(t), \ldots, y_{n}(t)\right)^{T}$ is the unknown function defined on $[a-\tau, b], \varphi(t)=\left(\varphi_{1}(t), \ldots, \varphi_{n}(t)\right)^{T}$ is continuous on $[a-$ $\tau, a], P_{k}=\left[p_{i j}^{(k)}\right]_{i, j=1, \ldots, n}, k=1,2$, are constant matrices and $P_{1}$ is nonsingular, $\tau_{i}>0$ for $i=1, \ldots, k$ and $\tau=\min _{1 \leq i \leq k}\left\{\tau_{i}\right\}$.

It is easy to see that after dividing both sides of equation (1.1) by $\epsilon$, problem (1.1)-(1.2) reduces to problem (1.3)-(1.4).

As the numerical experiments showed, this new approach gives more accurate approximate solutions for many boundary value problems for delay differential equations when compared with other known methods. Its application requires a reduction of the original problem to a fixed-point problem, which in the case of boundary value problem for DDE can be done with the help of an 
auxiliary linear system of equations of the form $\boldsymbol{y}^{\prime}=\boldsymbol{B} y+\boldsymbol{v}$ (with properly written original boundary conditions). Then, using the integral form of the solution of this problem with $v$ as a parameter function we eliminate $y^{\prime}$ and $y$ from the original equation and arrive at the fixed-point problem with $v$ as the unknown. In the above-mentioned papers an approximation to the unknown fixed-point $v$ was searched in the form of piecewise linear function $v_{h}$. An approximate solution $y_{h}$ was recovered through the integral form of the solution to the above-mentioned linear problem and assumed the form of a cubic (or quadratic) spline. It was interesting for us whether we can apply this approach to solving boundary value problems for singularly perturbed DDEs with such a success and without additional difficulties which introduce the usage of nonuniform grids. It is well known that such problems are more difficult to deal with and require special treatment which includes Shishkin-type meshes or Bakhvalov-type meshes (see $[1,6,8]$ ). Preliminary numerical experiments showed that the approach used in $[2,3]$ fails to produce accurate results. So, we decided to replace the piecewise linear approximation of the fixed point $v$ with the cubic spline approximation. The numerical experiments demonstrate that the change resulted in satisfactory accuracy of the approximation and the comparison with the accuracy of other methods shows that for some problems (with not too small perturbation parameter $\epsilon$ ) the accuracy is better ${ }^{1}$.

\section{Reduction of Problem (1.1)-(1.2) to a Fixed-Point Problem}

As we noticed earlier, problem (1.1)-(1.2) reduces to problem (1.3)-(1.4) and, in the sequel, we will use notation (1.3)-(1.4) for the boundary value problem for second order singularly perturbed delay differential equation under consideration. Following [2] we use the standard substitutions $y_{1}=y, y_{2}=y_{1}^{\prime}$ and reduce the system (1.3)-(1.4) to the system

$$
\begin{aligned}
& \boldsymbol{y}^{\prime}(x)=\boldsymbol{F}(x, \boldsymbol{y}(x), \boldsymbol{y}(\cdot)), \\
& y_{1}(x)=\phi(x) \text { for } x \leq a, \quad y_{1}(b)=0
\end{aligned}
$$

with

$$
\boldsymbol{y}(x)=\left(\begin{array}{l}
y_{1}(x) \\
y_{2}(x)
\end{array}\right), \quad \boldsymbol{F}(x, \boldsymbol{y}(x), \boldsymbol{y}(\cdot))=\left(\begin{array}{c}
y_{2}(x) \\
f\left(x, y_{1}(x), y_{2}(x), y_{1}(\alpha(x))\right)
\end{array}\right) .
$$

Now, let $\boldsymbol{P}_{1}$ and $\boldsymbol{P}_{2}$ be defined as follows:

$$
\boldsymbol{P}_{1}=\left(\begin{array}{ll}
1 & 0 \\
0 & 0
\end{array}\right), \quad \boldsymbol{P}_{2}=\left(\begin{array}{ll}
0 & 0 \\
1 & 0
\end{array}\right)
$$

and assume that there exists a matrix $\boldsymbol{B}=\boldsymbol{B}(x), x \in[a, b]$, such that for the fundamental matrix $\mathcal{Y}$ of the system $\boldsymbol{y}^{\prime}=\boldsymbol{B} \boldsymbol{y}$, satisfying $\mathcal{Y}(a)=I$, the matrix $\boldsymbol{Q}=\boldsymbol{P}_{1}+\boldsymbol{P}_{2} \mathcal{Y}(b)$ is nonsingular.

\footnotetext{
1 it is customary to denote the perturbation parameter standing in front of $y^{\prime \prime}$ by the Greek letter $\epsilon$. We also use this letter but with different shape $\varepsilon$ in the context of the name of our method
} 
Let us consider the following auxiliary boundary value problem for the system of differential equations

$$
\begin{aligned}
& \boldsymbol{y}^{\prime}(x)=\boldsymbol{B} \boldsymbol{y}(x)+\boldsymbol{v}(x), \quad x \in[a, b], \\
& \boldsymbol{P}_{1} \boldsymbol{y}(a)+\boldsymbol{P}_{2} \boldsymbol{y}(b)=\boldsymbol{d}=\left(\begin{array}{l}
0 \\
0
\end{array}\right) .
\end{aligned}
$$

The solution to $(2.3)-(2.4)$ can be written

$$
\boldsymbol{y}(x)=\mathcal{Y}(x)\left[-\boldsymbol{Q}^{-1} \boldsymbol{P}_{2} \mathcal{Y}(b) \int_{a}^{b} \mathcal{Y}^{-1}(s) \boldsymbol{v}(s) d s+\int_{a}^{x} \mathcal{Y}^{-1}(s) \boldsymbol{v}(s) d s\right] .
$$

Denoting the right-hand side of $(2.5)$ by $(\boldsymbol{G} \boldsymbol{v})(x)$ we can write the solution to (2.3)-(2.4) in the form

$$
\boldsymbol{y}(x)=(\boldsymbol{G} \boldsymbol{v})(x)=\left(\begin{array}{l}
\left(g_{1} v\right)(x) \\
\left(g_{2} v\right)(x)
\end{array}\right)
$$

and reduce the problem $(2.1)-(2.2)$ to the following fixed point problem

$$
\boldsymbol{v}(x)=\boldsymbol{F}(x,(\boldsymbol{G} v)(x),(\boldsymbol{G} \boldsymbol{v})(\cdot))-\boldsymbol{B}(\boldsymbol{G} \boldsymbol{v})(x) .
$$

It is easy to check that taking $\boldsymbol{B}=\left(\begin{array}{ll}0 & 1 \\ 0 & 0\end{array}\right)$ we get $\mathcal{Y}=\left(\begin{array}{cc}1 & x-a \\ 0 & 1\end{array}\right)$ with $\operatorname{det} \boldsymbol{Q} \neq 0$ and the problem (2.5)-(2.7) takes the form

$$
\left(\begin{array}{l}
v_{1}(x) \\
v_{2}(x)
\end{array}\right)=\left(\begin{array}{c}
0 \\
f\left(x, y_{1}(x), y_{2}(x), y_{1}(\alpha(x))\right)
\end{array}\right), \quad x \in[a, b] .
$$

From (2.8) it follows immediately that $v_{1}(x) \equiv 0$ and denoting $v_{2}$ by $v$ we obtain

$$
v(x)=f\left(x, y_{1}(x), y_{2}(x), y_{1}(\alpha(x))\right)
$$

where

$$
\begin{aligned}
& y_{2}(x)=\left(g_{2} v\right)(x)=\int_{a}^{b} \frac{s-b}{b-a} v d s-\int_{a}^{b} v d s+\int_{a}^{x} v d s \\
& y_{1}(x)=\left(g_{1} v\right)(x)=\int_{a}^{x}(a-s) v d s+(x-a)\left(g_{2} v\right)(x) .
\end{aligned}
$$

It is clear that the operators $g_{1}$ and $g_{2}$ are linear. Let the spaces $C_{0}[a, b]=$ $\{u \in C[a, b]: u(a)=u(b)=0\}$ and $C[a, b]$ be equipped with the standard norm $\|u\|=\max _{a \leq t \leq b}|u(t)|$.

In [2] the following three lemmas have been proved.

Lemma 1. The operators $g_{1}: L^{2}[a, b] \rightarrow C_{0}[a, b]$ and $g_{2}: L^{2}[a, b] \rightarrow C[a, b]$ defined by the formulas (2.10) and (2.11) are continuous.

Now, let us define the operator $\bar{G}: C_{0}[a, b] \rightarrow C[a, b]$,

$$
(\bar{G} y)(x)= \begin{cases}\Phi(\alpha(x)) & \text { if } \alpha(x)<a \\ y(\alpha(x)) & \text { if } a \leq \alpha(x) \leq b .\end{cases}
$$


Lemma 2. The operator $\bar{G}: C_{0}[a, b] \rightarrow C[a, b]$ is continuous.

Next, remark that the right-hand side of (2.9) defines the operator $\mathcal{A}: L^{2}[a, b] \rightarrow C[a, b] \subset L^{2}[a, b]$, i.e.

$$
\begin{aligned}
(\mathcal{A} v)(x) & =f\left(x,\left(g_{1} v\right)(x),\left(g_{2} v\right)(x),\left(\bar{G}\left(g_{1} v\right)\right)(x)\right) \\
& =f\left(x, y_{1}(x), y_{2}(x), y_{1}(\alpha(x))\right) .
\end{aligned}
$$

We have the following lemma:

Lemma 3. The operator $\mathcal{A}: L^{2}[a, b] \rightarrow L^{2}[a, b]$ defined by (2.12) is continuous.

\section{Description of the Approximate Method and Its Con- vergence}

The description of the approximate method is given in $[2,3]$. The only difference consists in that instead of using piecewise linear functions to approximate the fixed point we use cubic splines. So, our example of convergent approximation of space $L$ is different and we cannot prove Theorem 3.1 given in [2]. As a result we use another approach to prove Theorem 3.2 stated in [2].

To make the paper at least partly self-contained we recall the following basic material from [2] (see also [3]).

\subsection{Notation and basic definitions}

Let $(L, d)$ be a metric space with a metric $d, M$ be an interval $\left(0, h_{0}\right], h_{0}>0$, or a sequence of positive real numbers convergent to zero, $\left\{S_{h}: h \in M\right\}$ be a family of spaces of grid functions defined for each $h$ on a finite set of grid points from a compact set $K,\left\{r_{h}: h \in M, r_{h}: L \longrightarrow S_{h}\right\}$ be a family of operators mapping $L$ onto $S_{h}$ and $\left\{p_{h}: h \in M, p_{h}: S_{h} \longrightarrow L\right\}$ be a family of operators mapping $S_{h}$ into $L$.

Remark 1. In the sequel, speaking of an arbitrary family of sets $Q_{h}$ or transformations $q_{h}$ we shall mean the families $\left\{Q_{h} \mid h \in M\right\}$ or $\left\{q_{h} \mid h \in M\right\}$ respectively.

Definition 1. We shall say that the family of spaces $S_{h}$ and the families of operators $r_{h}$ and $p_{h}$ define a convergent approximation of $L$ if for any $x \in L$ the condition

$$
d\left(p_{h} r_{h} x, x\right) \underset{h \rightarrow 0}{\longrightarrow} 0
$$

holds.

Definition 2. We shall say that the family $L_{M}=\left\{x^{h} \in L: h \in M\right\}$ of functions in $L$ is compact in $L$ with $h \rightarrow 0$ if from any sequence $x^{h_{n}}, x^{h_{n}} \in L_{M}$, $h_{n} \rightarrow 0$, a subsequence convergent in $L$ can be chosen.

Now, let $\varepsilon \geq 0$ be a given real number.

Definition 3. We shall say that $x \in L$ is an $\varepsilon$-fixed-point of $\mathcal{A}: L \rightarrow L$ if

$$
d(x, \mathcal{A} x) \leq \varepsilon .
$$




\subsection{The main theorem and corollaries, an approximate method for a fixed-point problem}

\section{Theorem 1. If}

a) operator $\mathcal{A}$ is continuous in $L$,

b) the family of spaces $S_{h}$ and the families of operators $r_{h}$ and $p_{h}$ define a convergent approximation of $L$,

then $\mathcal{A}$ possesses at least one fixed-point if and only if there exists a nonnegative function $\varepsilon(h), \varepsilon(h) \rightarrow 0$ for $h \rightarrow 0$ such that the operator $\mathcal{A}$ possesses $\varepsilon(h)$-fixed-points $p_{h} x_{h}$ and the family $\left\{p_{h} x_{h} \mid h \in M\right\}$ is compact with $h \rightarrow 0$.

\section{Corollary 1. If}

(i) the operator $\mathcal{A}: L \longrightarrow L$ is continuous and has a unique fixed-point $x$,

(ii) the family of spaces $S_{h}$ and the families of operators $r_{h}$ and $p_{h}$ define a convergent approximation of $L$,

(iii) for $c_{h}=d\left(0, p_{h} r_{h} x\right)$ a sequence $\left(p_{h} x_{h}\right)$ satisfying the condition

$$
d\left(p_{h} x_{h}, \mathcal{A} p_{h} x_{h}\right)=\varepsilon_{c}(h) \stackrel{\text { def }}{=} \min _{\left\{y_{h}: d\left(0, p_{h} y_{h}\right) \leq c_{h}\right\}} d\left(p_{h} y_{h}, \mathcal{A} p_{h} y_{h}\right)
$$

is compact with $h \rightarrow 0$,

then

$$
\lim _{h \rightarrow 0} p_{h} x_{h}=x .
$$

Corollary 1 suggests the following approximate method for a fixed-point problem with a continuous operator $\mathcal{A}: L \longrightarrow L$, which we will call the $\varepsilon(h)$ approximate method:

1. Choose such families of spaces $S_{h}$ of grid functions $u_{h}=\left(u_{1}, u_{2}, \ldots, u_{n(h)}\right)$ defined on finite sets of grid points $\left(x_{1}, x_{2}, \ldots, x_{n(h)}\right)$ in a given compact set $K$ and operators $p_{h}$ and $r_{h}$ that

(a) they define a convergent approximation of $L$,

(b) family of $p_{h} x_{h}$ satisfying $d\left(p_{h} x_{h}, \mathcal{A} p_{h} x_{h}\right) \longrightarrow 0$ if $h \rightarrow 0$ is compact with $h \rightarrow 0$,

(c) the functions

$$
q_{h}\left(u_{1}, u_{2}, \ldots, u_{n(h)}\right)=d\left(p_{h} u_{h}, \mathcal{A} p_{h} u_{h}\right)
$$

are continuous with respect to the variables $u_{1}, u_{2}, \ldots, u_{n(h)}$.

2. Choose large enough constant $c$ and small enough parameter $h$ and find an $\varepsilon_{c}(h)$ fixed-point of $\mathcal{A}$ by minimization of $q_{h}$ on the (compact in $S_{h}$ ) closed ball $B\left(0_{h}, c\right)$ of radius $c$ centered at $0_{h}$, where $0_{h}=(0, \ldots, 0)$ is the zero function in $S_{h}$.

Then $p_{h} x_{h}$ approximates the fixed-point $x$ of $\mathcal{A}$ because $p_{h} x_{h} \rightarrow x$ if $h \rightarrow 0$. 
Remark 2. It follows from the proof of Theorem 1 (see [2]) that this method can be applied to the problems with a unique fixed-point if operator $\mathcal{A}$ is continuous only in some neighborhood of the fixed-point and if the initial approximation to an $\varepsilon_{c}(h)$ fixed-point is taken close enough to the fixed-point of $\mathcal{A}$.

\subsection{An example of a convergent approximation and other auxiliary theorems}

Like in [2], let

$$
L_{n}^{2}[a, b]=\underbrace{L^{2}[a, b] \times \cdots \times L^{2}[a, b]}_{n \text { times }}
$$

be the metric space of square integrable on $[a, b]$ vector functions with the metric $d$ induced by the norm $\|\cdot\|_{2}^{(n)}$ defined for $v \in L_{n}^{2}[a, b]$ by putting:

$$
\|v\|_{2}^{(n)}=\left(\sum_{i=1}^{n}\left\|v^{i}\right\|_{2}^{2}\right)^{\frac{1}{2}}, \quad \text { where }\left\|v^{i}\right\|_{2}=\left(\int_{a}^{b}\left|v^{i}(s)\right|^{2} d s\right)^{\frac{1}{2}} .
$$

Now, let on $[a, b]$ be defined a family of grids $N_{H}\left(H=2 h, h=\frac{b-a}{2 N}, N=\right.$ $1,2, \ldots)$ with grid points $x_{k}=a+k H, k=0,1, \ldots, N$. Denote the set of $h$ by $M$ and for $h \in M$ let

$$
S_{h}^{n}=S_{h}^{n}[a, b]=\underbrace{S_{h}[a, b] \times \cdots \times S_{h}[a, b]}_{n \text { times }}
$$

be a linear space (over $\mathbb{R}$ ) of grid functions defined on the grid $N_{H}$.

We define the extension operators $p_{h}: S_{h}^{n} \rightarrow L_{n}^{2}[a, b]$ by the formula

$$
p_{h} v_{h}=\left(\bar{p}_{h} v_{h}^{1}, \ldots, \bar{p}_{h} v_{h}^{n}\right),
$$

where $\bar{p}_{h} v_{h}^{i}$, for a fixed $h$, is the cubic interpolating spline whose knots coincide with the grid points $N_{H}$ and its values at the knots are the same as the values of the grid function at these knots. For the uniqueness of the cubic spline two more conditions are needed. So, we impose the following additional conditions

$$
\begin{aligned}
\bar{p}_{h} v_{h}^{i^{\prime}}(a) & =\left(-11 v_{h}^{i}\left(x_{0}\right)+18 v_{h}^{i}\left(x_{1}\right)-9 v_{h}^{i}\left(x_{2}\right)+2 v_{h}^{i}\left(x_{3}\right)\right) /(6 H), \\
\bar{p}_{h} v_{h}^{i^{\prime}}(b) & =\left(11 v_{h}^{i}\left(x_{N}\right)-18 v_{h}^{i}\left(x_{N-1}\right)+9 v_{h}^{i}\left(x_{N-2}\right)-2 v_{h}^{i}\left(x_{N-3}\right)\right) /(6 H) .
\end{aligned}
$$

We also extend the domain of the function $v \in L_{n}^{2}[a, b]$ by putting

$$
v(s)=0 \quad \text { if } s \notin[a, b] .
$$

Now, for a fixed $h$ define the restriction operator $r_{h}: L_{n}^{2}[a, b] \rightarrow S_{h}^{n}$ by putting $r_{h} v=\left(\bar{r}_{h} v^{1}, \ldots, \bar{r}_{h} v^{n}\right)$, where

$$
\bar{r}_{h} v^{i}\left(x_{k}\right)=\tilde{v}^{1}\left(x_{k}\right)=\frac{1}{2 h} \int_{-\infty}^{\infty} \omega_{h}\left(x_{k}, s\right) v^{i}(s) d s .
$$

The integral appearing in this formula is a Lebesgue integral and the function $\omega_{h}(x, s)$ is an averaging kernel (for details on averaging kernels see [4]). 
Now, we have to show that the family of spaces $S_{h}^{n}$ and the families of operators $p_{h}$ and $r_{h}$ define a convergent approximation of $L_{n}^{2}[a, b]$. Let $\tilde{v}_{h}=$ $\left(\tilde{v}_{h}^{1}, \ldots, \tilde{v}_{h}^{n}\right)$ be the average of the function $v$ obtained by applying the averaging kernel $\omega_{h}(x, s)$.

Theorem 2. For each $v \in L_{2}^{(n)}[a, b]$

$$
d\left(p_{h} r_{h} v, v\right) \underset{h \rightarrow 0}{\longrightarrow} 0
$$

i.e. the family of spaces $S_{h}^{n}$ and the families of operators $p_{h}$ and $r_{h}$ define a convergent approximation of $L_{2}^{(n)}[a, b]$.

Proof. We have

$$
\left\|p_{h} r_{h} v^{i}-v^{i}\right\|_{2} \leq\left\|p_{h} r_{h} v^{i}-\tilde{v}_{h}^{i}\right\|_{2}+\left\|\tilde{v}_{h}^{i}-v^{i}\right\|_{2} .
$$

To prove our theorem it is enough to prove that both terms on the right-hand side of inequality (3.6) converge to 0 as $h \rightarrow 0$. The convergence to 0 as $h \rightarrow 0$ of the second term on the right-hand side of inequality (3.6) follows from the following lemma proved in [4] (with $D=[a, b]$ and $p=2$ ).

Lemma 4. If $h \rightarrow 0$ then $\tilde{v}_{h} \rightarrow 0$ in the space $L^{p}(D)$.

To prove the convergence to 0 as $h \rightarrow 0$ of the first term on the right-hand side of inequality (3.6) we will use the results of [7, pages 35, 36]. Namely, the following corollary of the earlier proved theorem [7, Theorem 7.6, page 35] given in the form of an example and which (up to the notation adopted to our needs) reads

"As an application of Theorem 7.6, consider the case of cubic splines on a uniform partition $N_{H}$, where $N_{H}$ has at least four knots. Then, given $f \in C^{k}[a, b], 0 \leq k<4$ and $s$ be the unique cubic spline such that

$$
\begin{aligned}
& s\left(x_{i}\right)=f\left(x_{i}\right), \quad 0 \leq i \leq N, \\
& D s^{\prime}(a)=\left(-11 f\left(x_{0}\right)+18 f\left(x_{1}\right)-9 f\left(x_{2}\right)+2 f\left(x_{3}\right)\right) /(6 H), \quad(3.7) \\
& D s^{\prime}(b)=\left(11 f\left(x_{N}\right)-18 f\left(x_{N-1}\right)+9 f\left(x_{N-2}\right)-2 f\left(x_{N-3}\right)\right) /(6 H) .
\end{aligned}
$$

Then

$$
K H^{k-j} \omega\left(D^{k} f, H\right) \geq \begin{cases}\left\|D^{j}(f-s)\right\|_{L_{\infty}[a, b]}, & 0 \leq j \leq k, \\ \left\|D^{j} s\right\|_{L_{\infty}[a, b]}, & \text { if } k<j \leq 3,\end{cases}
$$

where $D$ stands for the derivative operator and $\omega$ is the module of continuity of $f$."

yields the claimed convergence.

It follows from the corresponding lemma proved in [4], that $\tilde{v}$ is continuous on $[a, b]$ (and consequently uniformly continuous) and the inequality (3.9) can be applied to it at least for $k=0, j=0$ (and for larger $k$, if $v$ is smooth). So, our theorem is proved. 


\subsection{Convergence of the approximate method}

To show that the method described in Section 3.2 converges we have to prove that the family $p_{h} v_{h}$ obtained by minimization on a ball $B(0, c)$ in $S_{h}$ the functions $q_{h}$ defined by (3.5) is compact with $h \rightarrow 0$, i.e. the family $p_{h} v_{h}$ is bounded in $L^{2}[a, b]$ and

$$
\int_{a}^{b}\left|p_{h} v_{h}(x+t)-p_{h} v_{h}(x)\right|^{2} d x \underset{t \rightarrow 0}{\longrightarrow} 0
$$

uniformly with respect to all $p_{h} v_{h}$. It is clear that (3.10) is equivalent to $\left\|\left(p_{h} v_{h}\right)_{t}-p_{h} v_{h}\right\|_{2} \underset{t \rightarrow 0}{\longrightarrow} 0$, where for any $w \in L^{2}[a, b]$ we use the notation $w_{t}(x)=w(x+t)$. Now, we can formulate the lemma (cp. Lemma 4.4 in [2]).

Lemma 5. The family $p_{h} v_{h}$ satisfying

$$
\left\|p_{h} v_{h}-\mathcal{A} p_{h} v_{h}\right\|_{2}=\min _{u_{h} \in B(0, c)}\left\|p_{h} u_{h}-\mathcal{A} p_{h} u_{h}\right\|_{2}
$$

is compact with $h \rightarrow 0$.

Proof. Let $p_{h} v_{h}$ satisfy (3.11). To prove the boundedness of $\left\|p_{h} v_{h}\right\|_{2}$ we follow [10] and denote the cubic spline $p_{h} v_{h}$ by $s_{h}$ and write it in the form

$$
s_{h}(x)=f_{i-1} p_{i}(x)+f_{i} q_{i}(x)+\beta_{i-1} u_{i}(x)+\beta_{i} v_{i}(x) \text { for } x \in\left[x_{i-1}, x_{i}\right],
$$

with

$$
\begin{aligned}
& p_{i}(x)=\frac{\left(x-x_{i}\right)^{2}}{h^{3}}\left[h+2\left(x-x_{i-1}\right)\right], \quad q_{i}(x)=\frac{\left(x-x_{i-1}\right)^{2}}{h^{3}}\left[h-2\left(x-x_{i}\right)\right], \\
& u_{i}(x)=\frac{\left(x-x_{i}\right)^{2}\left(x-x_{i-1}\right)}{h^{2}}, \quad v_{i}(x)=\frac{\left(x-x_{i-1}\right)^{2}\left(x-x_{i}\right)}{h^{2}},
\end{aligned}
$$

where $s_{h}\left(x_{i}\right)=f_{i}, i=0,1, \ldots, N, s_{h}^{\prime}\left(x_{i}\right)=\beta_{i}, i=1,2, \ldots, N-1, \beta_{0}$ and $\beta_{N}$ are defined by formulas (3.7) and (3.8). Vector $\vec{\beta}=\left(\beta_{1}, \ldots, \beta_{N-1}\right)$ satisfies the system of equations $A \vec{\beta}=\vec{f}$ with $A=D+B$, where $D=4 \operatorname{diag}(1 / h), B$ is a symmetric tridiagonal matrix with $b_{i i}=0, b_{i \pm 1, i}=1 / h$ and $\vec{f}=\left(3\left(f_{2}-\right.\right.$ $\left.\left.f_{0}\right) / h^{2}-\beta_{0} / h, 3\left(f_{3}-f_{1}\right) / h^{2}, \ldots, 3\left(f_{N-1}-f_{N-3}\right) / h^{2}, 3\left(f_{N}-f_{N-2}\right)-\beta_{N} / h\right)$.

Now, we can use Theorem 2.1 from [10], and for $1 \leq p<\infty$ get the inequality

$$
\left\|A^{-1}\right\| \leq \frac{h}{2}
$$

The boundedness of $\left\|p_{h} v_{h}\right\|_{2}$ follows from the form of formula (3.12), formulas for $\beta_{0}, \beta_{N}$ and estimate of vector $\vec{\beta}$ obtained from inequality (3.13). The remaining part of the proof goes exactly like in the proof of Lemma 4.4 in [2]. 


\section{Numerical Experiments}

Example 1. Consider the following boundary value problem ( [5]):

$$
\begin{aligned}
& \epsilon^{2} y^{\prime \prime}(x)-2 y(x-\delta)-y(x)=1, \\
& y(x)=1 \text { for }-\delta \leq x \leq 0, \quad y(1)=0 .
\end{aligned}
$$

The maximum absolute errors $E^{N}=\max _{1 \leq i \leq N-1}\left|y_{i}^{N}-y_{2 i}^{2 N}\right|$ obtained by using the presented method and comparison with the absolute errors given in [5] are shown in Table 1.

Table 1. Errors $E^{N}: \epsilon=0.1, \delta=0.06$.

\begin{tabular}{cccccc}
\hline Method $\downarrow N \rightarrow$ & 100 & 200 & 300 & 400 & 500 \\
\hline Our method & 0.000106 & 0.000052 & 0.000027 & 0.000016 & 0.000007 \\
\hline Difference method [5] & 0.000327 & 0.000082 & 0.000036 & 0.000020 & 0.000013 \\
\hline
\end{tabular}

Figure 1 depicts the graphs of the solution of the problem and absolute errors for $N=500$.

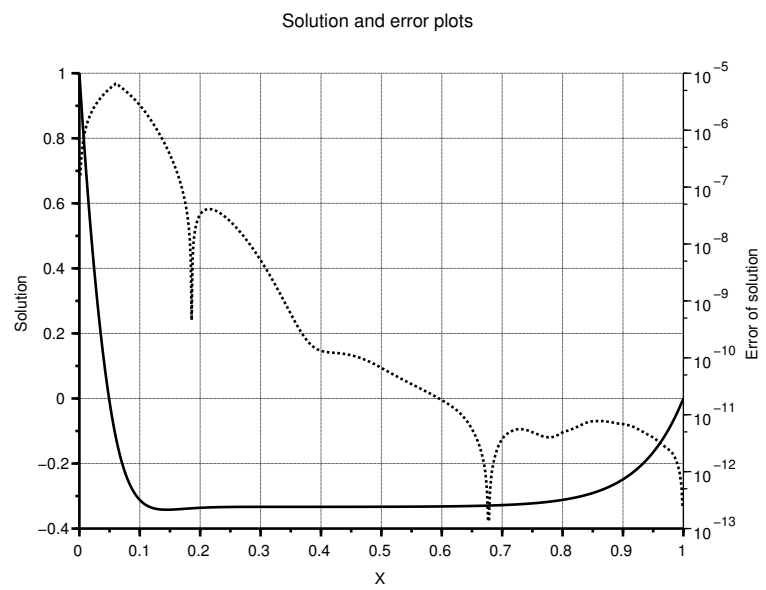

Figure 1. Numerical solution of problem (4.1) (solid line) and its absolute error (dotted line).

On the basis of the errors given in Table 1 we can conclude that the numerical average order of the proposed method is equal to 1.36 .

Example 2. Consider the following boundary value problem [8]:

$$
\begin{aligned}
& -\epsilon^{2} y^{\prime \prime}(x)+\frac{y-1}{2-y}+f(x)=0, \\
& y(0)=0, \quad y(1)=0,
\end{aligned}
$$


Table 2. Absolute errors for $\epsilon=2^{-6}$ and $N=512$.

\begin{tabular}{llllll}
\hline Method & $(H, S) \&(m H, S)$ & $(m H, B)$ & $(H, B)$ & $(m H, B$, var $q)$ & $(Z B, A B)$ \\
\hline Error & $3.6 \cdot 10^{-4}$ & $2.4 \cdot 10^{-8}$ & $7.4 \cdot 10^{-8}$ & $1.5 \cdot 10^{-7}$ & $7.5 \cdot 10^{-9}$ \\
\hline $\begin{array}{l}(H, S) \\
(m H, S)\end{array}$ & (Hermite scheme, Shishkin-type mesh) \\
$(m H, B)$ & (modified Hermite scheme, Shishkin-type mesh) \\
$(H, B)$ & (Hermite scheme, Bakhvalov-type mesh) \\
$(m H, B, \operatorname{var} q)$ & (modified Hermite scheme, Bakhvalov-type mesh, variable $q)$ \\
$(Z B, A B)$ & uniform mesh used by the authors of the paper
\end{tabular}

where $f$ is chosen in such a way that the exact solution is:

$$
y_{\epsilon}(x)=1-\frac{\exp (-x / \epsilon)+\exp ((x-1) / \epsilon)}{1+\exp (-1 / \epsilon)} .
$$

The maximum absolute errors obtained by using the presented method and comparison with errors given in [8] are shown in Table 2.

In Figure 2 we present the graph of the solution of (4.2) (solid line, the $y$ axis on the left), the graph of the absolute error of the solution of (4.2) (dotted line, the $y$-axis on the right) and the graph of the absolute error of the first derivative of the solution of (4.2) (dashed line, the $y$-axis in the center).

Solution and error plots

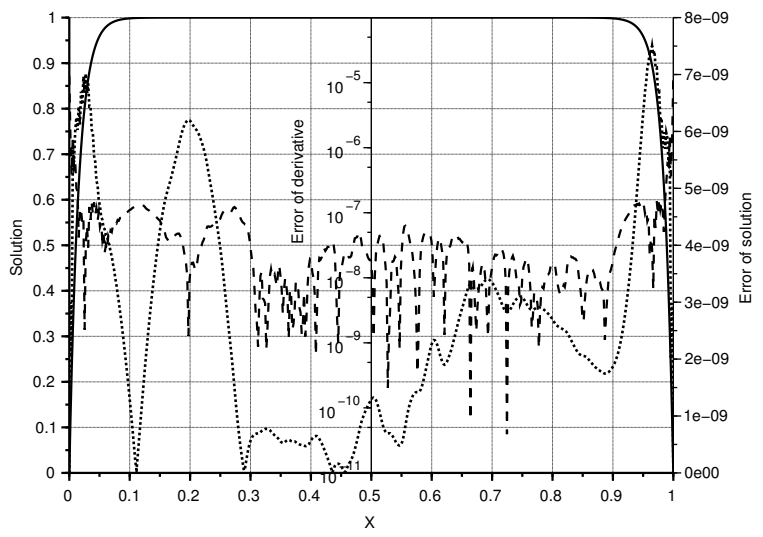

Figure 2. Numerical solution of problem (4.2) (solid line), absolute error of solution (dotted line) and absolute error of the first derivative (dashed line). 


\section{Conclusions}

We presented a fixed-point approach to solve boundary value problem for second order singularly perturbed delay differential equation (1.1)-(1.2). Using the auxiliary problem (2.3)-(2.4) we reduce problem (2.1)-(2.2) to a fixed point problem. The proposed method can be extended in a way similar to that used in [3] so that it can be applied to systems of singularly perturbed delay differential equations with general linear boundary conditions. The developed method can be applied to linear and nonlinear problems assuming only the existence and uniqueness of solution and continuity of the right-hand side of the differential equation. We showed that in the case considered in this paper the sought fixed-point $v$ is the second derivative $y^{\prime \prime}$ of the unknown function $y$. The function $y$ and its derivative $y^{\prime}$ are given by (2.10) and (2.11) respectively. So, in addition to the solution $y$ at the same time we obtain at no cost $y^{\prime \prime}$ and at a low cost $y^{\prime}$, which is one of the advantages of the method. In the development of solution methods for the problems under consideration there are two difficulties: the first one is caused by the presence of a delay term and the second one is due to the singular perturbation parameter. To cope with the first difficulty it was enough to approximate the fixed-point of the suitably chosen operator with a piecewise linear function. To cope with the presence of boundary layers caused by the singular perturbation parameter we employed the cubic spline approximation of the fixed-point. The numerical experiments show that for not too small singular perturbation parameters the obtained approximate solutions are accurate and comparable to those obtained by other robust methods or even slightly better. However, in the case of small singular perturbation parameter, even though formula (2.10) for $v$ replaced with a cubic spline produces a quintic spline, we cannot obtain a satisfactory accuracy of an approximate solution. Another drawback of the proposed method is that it uses minimization of $L^{2}$ norm of a residuum (see, for example, formula (3.11)) and converts linear problems into nonlinear problems. So, in the case of a specific form of the right-hand side of equation (1.1) other (direct and specific oriented) methods usually will be more time-effective.

\section{Acknowledgements}

The authors wish to express their gratitude to anonymous referees for very useful comments, suggestions and corrections which improved the presentation of this paper.

\section{References}

[1] N.S. Bakhvalov. Towards optimization of methods for solving boundary value problems in presence of a boundary layer. Zh. Vychisl. Mat. i Mat. Fiz., 9:841859, 1969. (in Russian)

[2] Z. Bartoszewski. A new approach to numerical solution of fixed-point problems and its application to delay differential equations. Appl. Math. Comput., 215:4321-4332, 2010. http://dx.doi.org/10.1016/j.amc.2009.12.058. 
[3] Z. Bartoszewski. Solving boundary value problems for delay differential equations by a fixed-point method. J. Comput. Appl. Math., 236:1576-1590, 2011. http://dx.doi.org/10.1016/j.cam.2011.09.021.

[4] L.V. Kantorovich and G.P. Akilov. Functional Analysis, 2nd ed. Pergamon Press, Oxford-Elmsford, New York, 1982.

[5] M.K.Kadalbajoo and K.K. Sharma. Numerical treatment of boundary value problems for second order singularly perturbed delay differential equations. Comput. Appl. Math., 24(2):151-172, 2005.

http://dx.doi.org/10.1590/S0101-82052005000200001.

[6] G.I. Shishkin. Grid approximation of singularly perturbed parabolic equations with internal layers. Sov. J. Numer. Anal. Math. Model., 3:392-407, 1988. http://dx.doi.org/10.1515/rnam.1988.3.5.393.

[7] B.K. Swartz and R.S. Varga. Error bounds for spline and l-spline interpolation. J. Approx. Theory, 6(1):6-49, 1972. http://dx.doi.org/10.1016/0021-9045(72)90079-2.

[8] R. Vulanović. Fourth order algorithms for a semilinear singular perturbation problem. Numer. Algorithms, 16:117-128, 1997. http://dx.doi.org/10.1023/A:1019187013584.

[9] Y. Zhang, D.S. Naidu, C. Cai and Y. Zou. Singular perturbations and time scales in control theories and applications: an overview 2002-2012. Int. J. Inf. Syst. Sci., 9(1):1-36, 2014.

[10] Z. Zhang and C.F. Martin. Convergence and Gibbs' phenomenon in cubic spline interpolation of discontinuous functions. Numer. Algorithms, 87:359-371, 1997. http://dx.doi.org/10.1016/S0377-0427(97)00199-4. 